\title{
Design of Bionic Bamboo Steel Bicycle
}

\author{
Tao Huang ${ }^{1, a}$, Yanqin Gao ${ }^{1, b}$, Maohuang $\mathrm{Xu}^{1, \mathrm{c}}$ and Mingming $\mathrm{Zhu}^{1, \mathrm{~d}}$ \\ ${ }^{1}$ School of Architecture and design, Southwest Jiaotong University, Chengdu 610000, China \\ adesignhuangtao@163.com, ${ }^{b}$ lanxinjoy0116@163.com, ${ }^{\mathrm{C}} 774603624 @ Q Q . c o m$, \\ d827083432@QQ.com
}

Keywords: bamboo steel; bionics; bicycle; design

Abstract: Bamboo steel is composed of recyclable sinocalamus affinis with rich resources in China, environmentally friendly phenolic resin and moisture. The high-performance multi-purpose bamboo-based fiber composite processed with multiple technologies does not require special care and maintenance in use. Based on good elasticity and toughness, high intensity, high weather fastness, fire resistance, carbon-negative environmental protection and long service life of bamboo steel, this paper starts from the feasibility of its application to bicycle design and designs panda bamboo steel bicycle in combination with the principle of bionic design. This paper analyzes design elements of panda bamboo steel bicycle and further illustrates its realizability, practicability, vitality and feature of Sichuan culture through the verification of model test program and analysis on model dimension.

\section{Introduction}

Bionic design integrates its form into product design perfectly based on the correlation between the cognition of biological features and elements of design objects so as to realize breakthrough and innovation, imply the beauty and scientificity of natural biology in design and meanwhile manifest humanistic color and regional characteristics ${ }^{[1]}$.The application of bionics to appearance design of vehicles such as automobile and motorcycle is common, but its application to appearance design of bicycle is rare. With multi-purpose bamboo-based fiber composite characterized by good elasticity and toughness, weather fastness, fire resistance and high intensity- bamboo steel as the main material, this paper designs bionic bamboo steel bicycle. Special care and maintenance are not required for bamboo steel in use. It can effectively absorb carbon dioxide and a lot of hazardous substances in air. Characterized by high intensity, low carbon and environmental protection, high weather fastness, fire resistance, air purification and long service life, it is the best product for developing bamboo in place of steel.

On the one hand, panda bamboo steel bicycle designed in this paper can inject new vigor into appearance design of current bicycle, promote the exploration of new materials and improve the ability of autonomous design of domestic bicycle. On the other hand, Sichuan is the hometown of panda, while the material of bamboo steel comes from bamboo. Their combination manifests the integration and unification of Sichuan natural landscape, humanities and product design.

\section{Feasibility of design of bamboo bicycle}

Bamboo steel (Fig.1) is a high-performance multi-purpose bamboo-based fiber composite with sinocalamus affinis as raw material. High performance and controllability of bamboo-based fiber composite is realized through fibering bamboo curtain preparation technology, uniform importing technology of phenolic resin, wide panel paving technology and molding and curing technology. This technology omits the traditional stripping process and can increase the use ratio of bamboo wood from current $20 \% \sim 50 \%$ to over $95 \%$. Its mechanical properties are as below: bending strength $\geq 350 \mathrm{MPa}$, tensile strength $\geq 360 \mathrm{MPa}$, compressive strength $\geq 140 \mathrm{MPa}$, elasticity modulus $\geq 30 \mathrm{GPa}$, boiled expansion rate $\leq 5 \%$, compression strength parallel to the grain $>180 \mathrm{MPa}$, tensile strength parallel to the grain $>300 \mathrm{MPa}$; its ratio of strength to weight exceeds that of glassfiber composite ${ }^{[2]}$. 

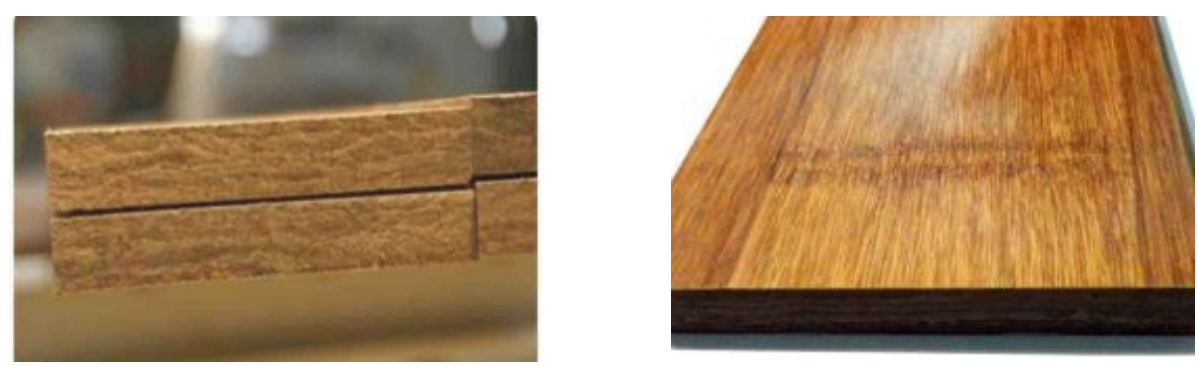

Fig.1 Section of bamboo steel material (left) and bamboo steel board (right)

The feasibility of bamboo steel bicycle can be manifested in multiple aspects. First, the material of bamboo steel has good feasibility, properties of which are better than bamboo wood and timber. Its static bending intensity, elasticity modulus and tensile strength are twice of those of common timber. Meanwhile, compared to bicycles made of other materials, bamboo steel bicycle has far lower energy consumption and great price advantage. In addition, bamboo steel is made of thermosetting resin of bamboo wood at high temperature and pressure. Therefore, it can be subject to integral moulding and can improve the stability of frame and the integrity of shape.

Then, bamboo steel has good feasibility in structure. In the preparation process of bamboo steel, the orientation of bamboo wood fiber is not disrupted. The original composite stiffness and structural stability of bamboo wood can be maintained. Moreover, it has good elasticity, toughness and rigidity, strong resistance to impact load and periodic fatigue damage and good resistance to shock and meets the requirement of bicycle structure for carrying capacity.

In addition, bamboo steel has good feasibility in appearance design. Bamboo steel has good coloring performance. It can be colored and painted and can meet color decoration requirements in appearance design. The material can reflect design concepts of low carbon, environmental protection, love for nature and sustainability. Its natural texture and shape can express the original nature of bamboo. Fashionable and concise shape can be designed. Meanwhile, it is easy to be processed and accepted by the public. Inherent properties of bamboo steel can be expressed in appearance, i.e. rigidity just opposite to gentle and elegant properties of bamboo, which gives people a sense of security ${ }^{[3]}$.

\section{Aesthetic property, practical property and superiority of panda bamboo steel bicycle}

The original intention of design is to design a series of bionic bamboo steel bicycle with bamboo steel as main material and various animals as reference. This paper conducts bionic bamboo steel bicycle design with panda as the case. Panda is the national treasure of China as well as one of the most precious animals in the world. Panda belongs to ursidae, which has big and fleshy body, round head, short tail and cute appearance. Its head and body are mostly chequered with black and white. Rostral side of its nose, eye socket, ears and limbs are in black. Its head and neck, body and tail are in white. As panda is a specific species in China and has special shape features and cultural value, it becomes a special Chinese element.

Panda bamboo steel bicycle designed in this paper is intended to be used at the gate of relevant scenic spots in Chengdu and as public-service bicycle. Panda bamboo steel bicycle can manifest Sichuan cultural features and meet requirements for low carbon and environmental protection. Bicycle frame made of bamboo steel has good toughness, high intensity and good damping effect. It has certain advantages. For wooden bicycle, timber has poorer weather fastness, fire resistance and intensity compared to bamboo steel and it will cause waste of resources for China and even the world with shortage of timber resources. For bicycles made of aluminium alloy, magnesium alloy and carbon fiber etc., such materials cannot regenerate or degrade quickly after abandonment ${ }^{[4]}$. However, bamboo steel is fast-growing and regenerative resource with good significance of environmental protection and sustainable development. 


\section{Design process of panda bamboo steel bicycle}

In terms of structure, a common bicycle includes over 20 parts such as frame, tire, pedal, brake and chain, basic parts of which are indispensable. In the design process of panda bamboo steel bicycle, this paper first conducts market investigation and analysis, conducts design orientation on this basis, implements program design, optimization and screening based on the structure and working principle of bicycle and the analysis on each design element and finally conducts man-machine analysis, detail analysis and mechanical test analysis on the final program.

\section{Analysis on basic design elements of panda bamboo steel bicycle}

In terms of functional element, it can travel safety and steadily and meet basic requirements of riding. Meanwhile, it has the significance of environmental protection and health. Bamboo steel material and non-coating orientation can manifest the significance of environmental protection and recoverability. In addition, as a healthier tool for riding instead of walk, bicycle is a fashion as well as advocation of health. In terms of man-machine element, the design of basic dimensions follows the standard specified in the Bicycle Volume of Chinese Light Industry Standard and optimal design is conducted in combination with ergonomics ${ }^{[5]}$. In terms of shape, the processing technology of bamboo steel is mainly considered. A bicycle conforming to the lifestyle and aesthetic habits of modern people, having common design language of modern product design and manifesting new elements of regional culture is designed with bionic style design method.

\section{Test program for model structure of panda bamboo steel bicycle}

Bionic design of shape of panda bicycle is conducted based on preliminary market investigation and design orientation, analysis on basic elements of panda bamboo steel bicycle, features of processing technology of bamboo steel material, morphological features of panda (Fig.2) and observation of its daily posture and body joints. While respecting objective aesthetic law, this paper uses advanced and environmentally friendly bamboo steel material for design, which meets basic requirements of product design and has originality in concept, thinking, method, manifestation and use. Model test combining appearance and structure is conducted after preliminary sketch conceiving and screening. The specific program is shown as below:
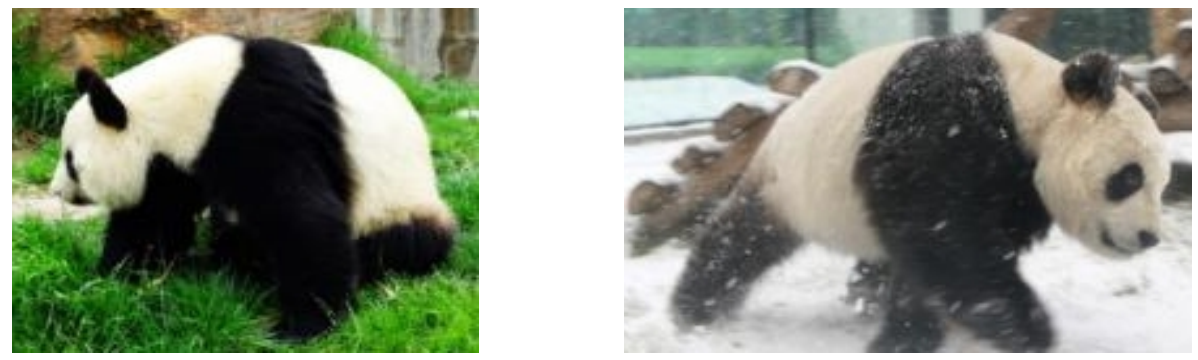

Fig.2 Daily life shape of panda

The inspiration of program I comes from the cute appearance of panda and the bicycle shape gives people a decorous feeling. The main frame is designed with the same posture and two structural forms. As is shown in Fig.3a, the first main frame has one-piece structure, in which the head and body are integrated and the head cannot be rotated. Two pieces in the front fork are separated with a big angle. Therefore, the head will not be interfered during turning. As is shown in Fig. 3b, the second main frame has one-piece structure, in which the head and body are two separate parts. The head can rotate with the front fork of bicycle. As is shown in Fig.3c, the third main frame has two-piece structure, in which the head and body are integrated and the head cannot be rotated. 


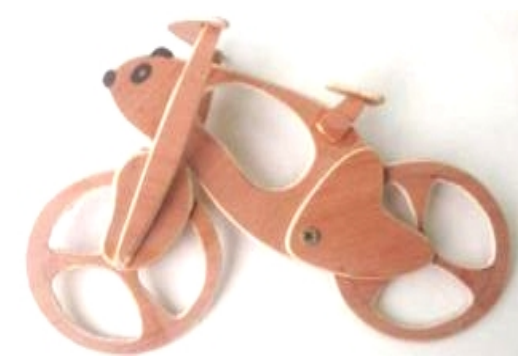

a

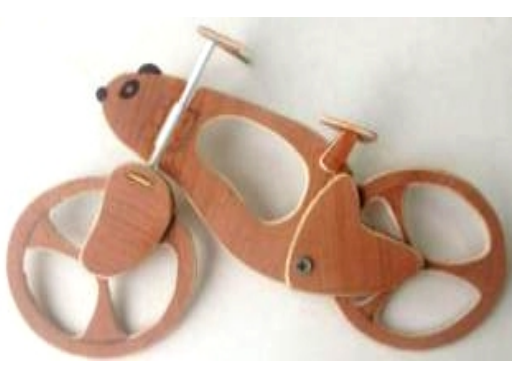

b

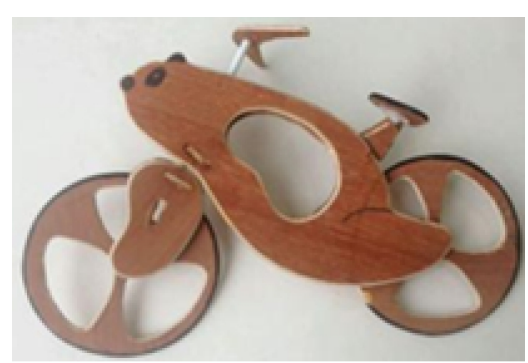

c

Fig.3 Test program for model structure in program I

Program II imitates the cute shape of panda. The bicycle designed gives people a sense of flexibility. As is shown in Fig.4a, the first main frame has one-piece structure, in which the head and body are two separate parts and the head can rotate with the front fork of bicycle. As is shown in Fig.4b, the second frame has two-piece structure, in which the head and body are integrated and the head cannot rotate with the front fork.

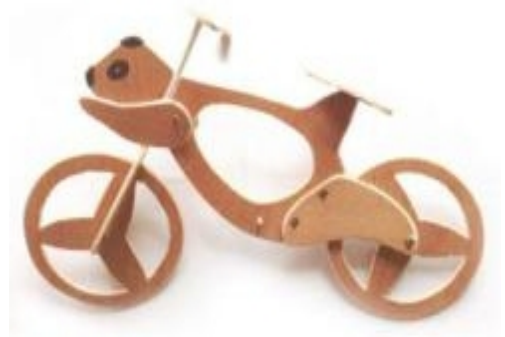

a

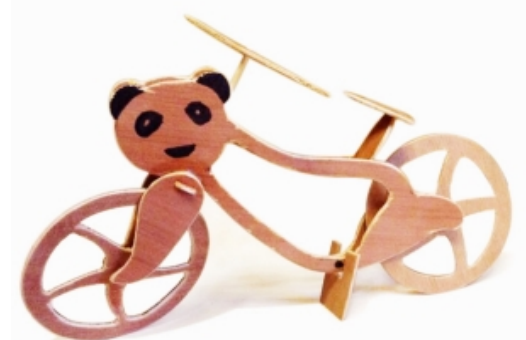

b

Fig.4 Test program for model structure in program II

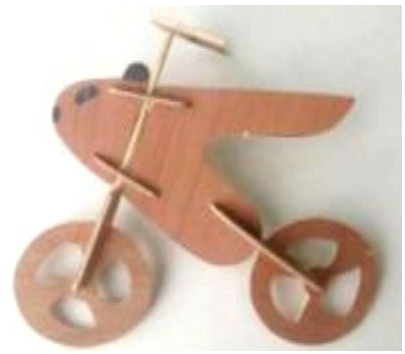

Fig.5 Test program for model structure in program III

As is shown in Fig.5, program III imitates morphological features of panda and conducts abstract bionic design of panda appearance. The frame is highly integrated and easy for processing and volume production. The main frame has one-piece structure, in which the head and body are integrated and the head cannot be rotated.

\section{Size structure and design process of panda bamboo steel bicycle}

To achieve the stability of panda bamboo steel bicycle, bamboo steel material is used for main frame parts such as frame, front fork, back form and handlebar and material with high stability that can stand market test is used for key parts of mechanical connection. Meanwhile, the bicycle should be easy to repair, have reliable performance and novel appearance and fully reflect leisure and interestingness of bionics. Bamboo steel can be subject to surface coating. However, this design does not consider color coating in the final effect so as to restore the real look of this ecological material and manifest its property of environmental protection and beauty of texture. In terms of consumer orientation, this product is intended to use as public-service bicycle and in relevant scenic spots in Sichuan for leisure and entertainment. It strives to manifest characteristics of panda and conform to regional culture of Sichuan. Fig.6a shows a picture of hand-made panda bamboo steel bicycle; Fig.6b shows the schematic diagram of riding; Fig.7 shows appearance and the name of each part of the product; 


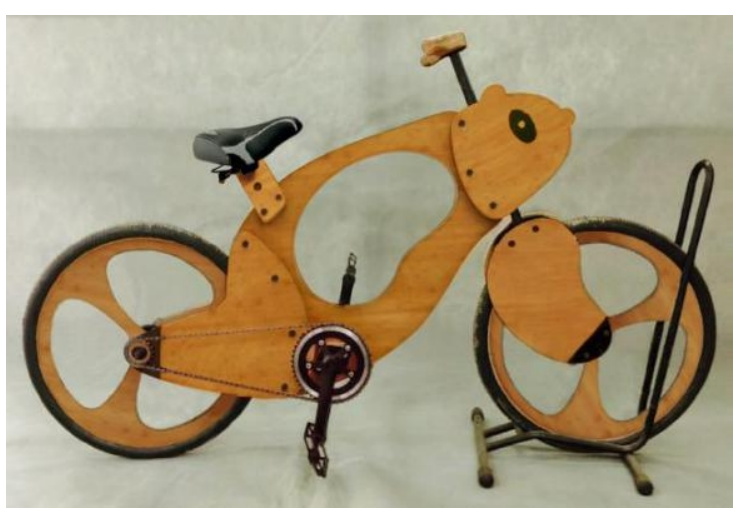

a Picture of handmade panda bamboo steel bicycle

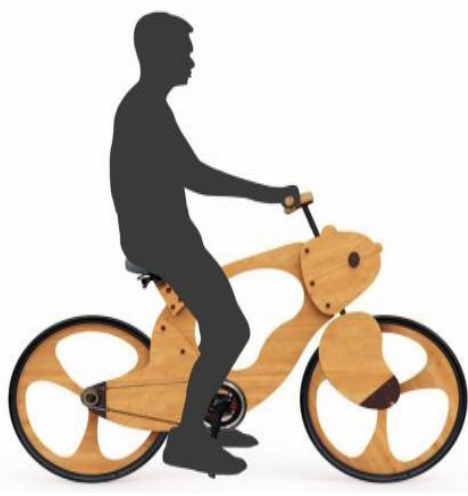

b Schematic diagram of riding

Fig.6 Picture of handmade panda bamboo steel bicycle

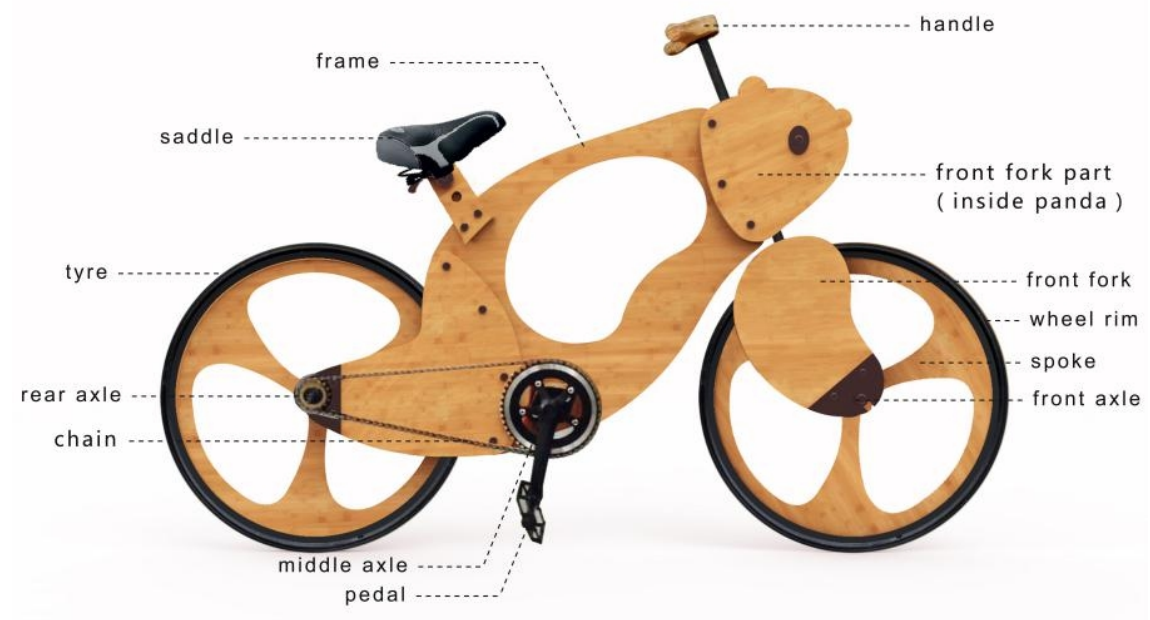

Fig.7 Appearance and name of each part of panda bamboo steel bicycle

\section{Overall dimension design of panda bamboo steel bicycle}

Appearance design of frame is conducted based on various postures of panda under different states, the result of investigation on users' favor and specific service environment and usage pattern of the bicycle. Meanwhile, consideration is given to technological properties of bamboo steel material and the influence of processing complexity and weight of frame on the overall strength and properties etc. ${ }^{[6]}$ The design of overall dimensions is completed based on the thought mentioned above and model test in combination with industrial standard of bicycle design in the Bicycle Volume of Chinese Light Industry Standard. See Fig.8.

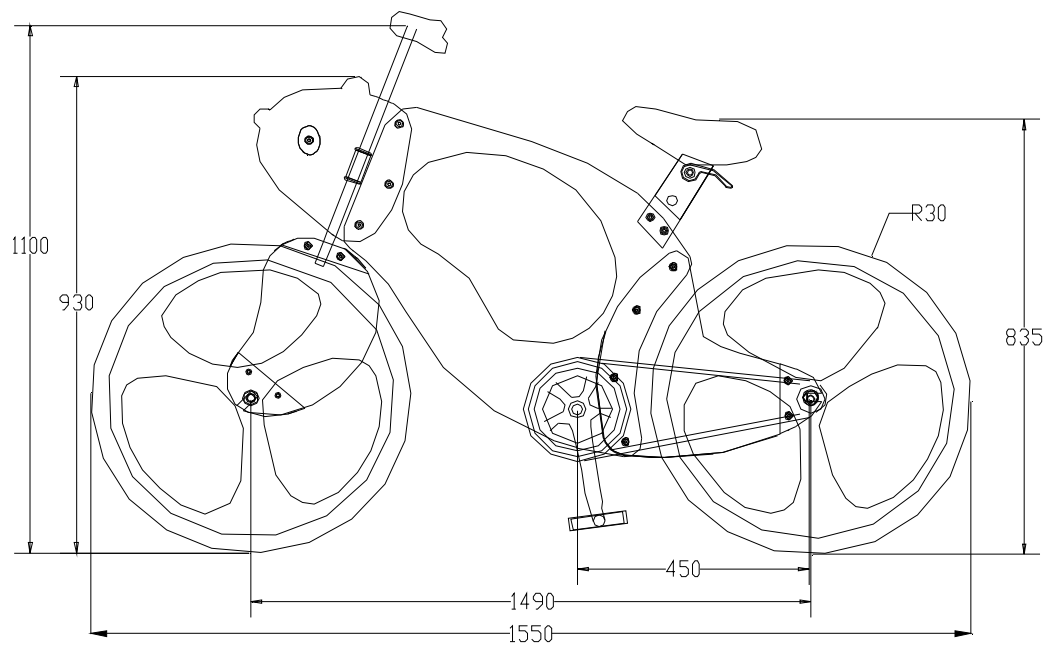

Fig.8 Dimensions of panda bamboo steel bicycle (unit:mm) 


\section{Design of detail size}

For detail size of frame of panda bamboo steel bicycle, see Fig.8. For its head size, see Fig.9a. For the size of foreleg, see Fig.9b. For the size of body, see Fig.9c. For the size of rear leg, see fig9d. Fig.8 shows the appearance of rim. As it concerns the riding performance of bicycle, cutter ring is used according to the final test result, which has good stability and is not easy to corrode and deform. According to the region for later use and riding requirements of the public, its size is defined as 24 inch. The spoke only has a beautiful effect. Two alternatives are considered: first, the use of bamboo steel material and integrated body design; second, purchasing of existing antirust spoke as an alternative to the first program. According to the standard specified in the Bicycle Volume of Chinese Light Industry Standard, 24 inch antirust or stainless steel chain is bought for use.

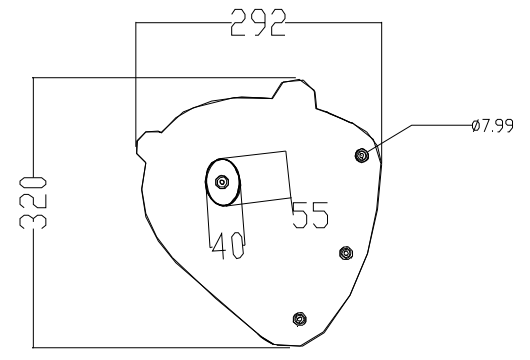

a

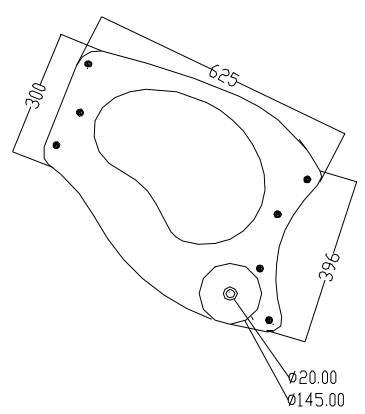

$\mathrm{c}$

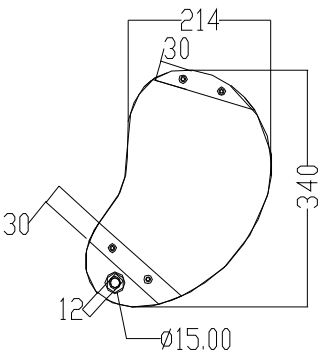

$\mathrm{b}$

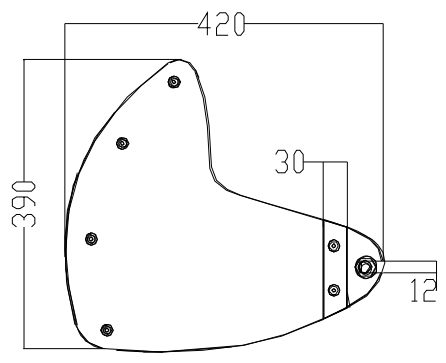

d

Fig.9 Detail drawing(unit:mm)

Metal material is used as carrier at the joint of front, middle and rear axles. The size of connecting shaft and bamboo steel body follows the Bicycle Volume of Chinese Light Industry Standard. Rear axle and flywheel are key parts of the bicycle. Coupling flywheel must be used for common non-variable speed bicycles. Finished products are bought based on service environment, usage pattern, design size and linkage structure. As front fork and fork attachment involve the conjunction and connection of panda head and body and front fork shape (foreleg of panda), which are different from metal bicycle shape design, and considering that it can allow bicycle handlebar to rotate flexibly, this part is completed according to the respective design program. For handlebar and its grips, man-machine design is mainly considered. Finished product is used in wrist group. Other front fork attachments should be designed according to actual requirements, but their dimensions should follow existing standard. See Fig.10. Brake equipment concerns the safety of riding. Therefore, existing products with good material property and high functionality should be bought for use. Front and rear wheels concern riding performance and are used in combination with rim. Therefore, existing 24 inch bicycle tires with slip resistance and low noise should be bought, which should match with the appearance. Man-machine size and material use should be considered for cushion. Generally, an anti-vibration device is installed below cushion so as to achieve comfortability during riding ${ }^{[7]}$. Considering the height of different people, two gears are set up for adjustment. Existing comfortable cushion should be bought for parts in contact with human body. See Fig.11. Slip resistance requirement is mainly considered for pedals. Existing matched grain products can be bought for use to achieve beauty and harmony. 


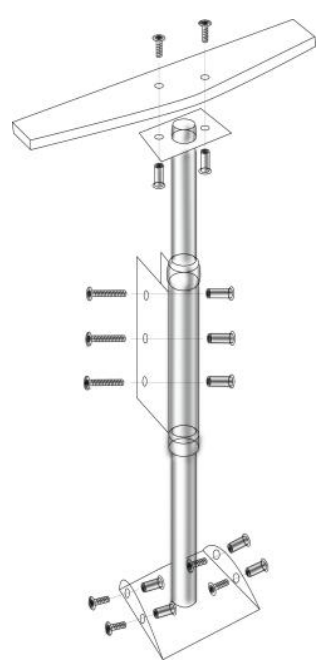

Fig.10 Explosive view of front fork attachments

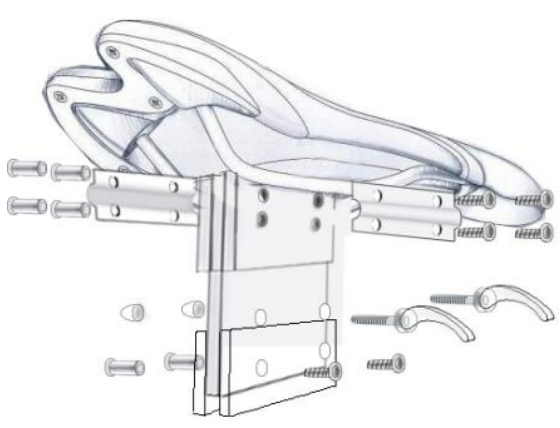

Fig.11 Explosive view of saddle

\section{Conclusions}

There are diversified materials used in the bicycle. Each material has its advantages and disadvantages. To give full play to such materials, their properties and product use requirements should be considered. Starting from the feasibility of bamboo steel bicycle, this paper designs panda bamboo steel bicycle based on the principle of bionic design. The material of bamboo steel bicycle is innovative. With good properties such as good elasticity and toughness and high intensity, bamboo steel meets material performance requirements of bicycle. Meanwhile, bamboo steel is regenerative resource with low carbon and environmental protection. Its has great significance in the development of new energy. Moreover, the appearance of panda bamboo steel bicycle caters for Sichuan culture. It has great significance for promoting local culture and creating regional name card.

As a new material, bamboo steel has a lot of superiorities in the application to bicycle and meanwhile improves the additional value of Chinese bamboo products. However, due to its weight and stress in texture processing, in-depth discussions should be made on its application to bicycle. For example, in terms of weight, its thickness can be reduced due to its high intensity and good toughness. In terms of texture, force bearing point should be parallel to the grain during processing. Due to the special grain of bamboo steel, force vertical to transverse grain direction will greatly impair its intensity and cause fracture. Corresponding better solutions should be put forward for existing problems so that bamboo steel can stand out among various materials of bicycle.

\section{References}

[1] Fen Li, Jianping Cai, Li Li. Discussions on Bionic Design of Product Shape [J]. Packaging Engineering, vol.11 2007.(In Chinese)

[2] Wentao Zhou et al. Study on Application of Bamboo Steel in Landscape Design [J]. Journal of Southwest China Normal University (Natural Science Edition), 2015.(In Chinese)

[3] Yanqin Gao. Study on Feasibility of Bamboo Steel Bicycle Frame. Design, 2015.7.(In Chinese)

[4] Shu Wang et al. Application of Bicycle Frame Material and Magnesium Alloy [J]. Materials Review, 2006.20(8).(In Chinese)

[5] Naying Zhang. Application of Ergonomics in Bicycle Product Design [J]. China Bicycle, vol.01 2001.(In Chinese)

[6] Translated by Yumin Xing. Bicycle Service Manual. Beijing: Light Industry Press, 1984.

[7] Haibo Huang, Yulan Ding. Man-machine Engineering Design of Bicycle Saddle. Journal of Engineering Graphics, 2005,3:106-110.(In Chinese) 